\title{
ik mideo dreuuet
}

\section{Notizen zum Profil der Frühmittelalterforschung und einige ketzerische Gedanken zum Gegenstand historischer Philologien}

\author{
Stephan Müller
}

Online publiziert: 20. Juni 2017

(C) Der/die Autor(en) 2017. Dieser Artikel ist eine Open-Access-Publikation.

Zusammenfassung Der vorliegende Essay versucht einige Besonderheiten der philologischen Frühmittelalterforschung gegenüber der philologischen Arbeit an Texten des Hoch- und Spätmittelalters zu profilieren. Die zentralen Thesen sind dabei: (1) Für das Frühmittelalter kann man auf ein Paradigma der Vollständigkeit setzen, während die Hoch- und Spätmittelalterforschung auf Praktiken der Selektion angewiesen ist. (2) Die Frühmittelalterforschung muss sich gegenüber ihren Gegenständen deutlicher und eindeutiger philologisch eingreifend positionieren, während die Hochund Spätmittelalterphilologie mit einer überzogenen Forderung nach philologischer Abstinenz zu kämpfen hat.

Schlüsselwörter Philologie $\cdot$ Prosopographie $\cdot$ Kodikologie · Handschriftenüberlieferung · Althochdeutsch · Frühmittelaltergermanistik

\section{ik mideo dreuuet}

Notes on the Profile of Early Medieval Research and some Heretical Thoughts on the Subject of Historical Philologies

\begin{abstract}
This essay strives to point out several characteristics which distinguish philological research on early medieval texts from methods used in working on high or late medieval literature. The key points are the following: (1) when dealing with the early Middle Ages, one can rely on a paradigm of completeness while research on high or late medieval texts depends on practices of selection. (2) Early medieval literature studies usually cannot refrain from reconstructing their texts by applying tools of textual criticism; in contrast, early and late medieval literary studies today are forced to struggle with excessive demands for philological restraint.
\end{abstract}

S. Müller $(\bowtie)$

Institut für Germanistik, Universität Wien, Wien, Österreich

E-Mail: stephan.mueller@univie.ac.at 
Keywords Philology · Prosopography · Codicology $\cdot$ Manuscript Transmission · Old High German · Early Germanic Studies

Das titelgebende Zitat dieses Essays stammt aus dem Hildebrandslied. Es ist das Ende des zwölften Verses, so wie er in der Handschrift steht. Hildebrand bittet seinen Sohn, den er noch nicht erkannt hat, ihm die Mitglieder seiner Sippe zu nennen. Hildebrand kenne die ganze Schar der Helden (irmindeot), wenn er nur einen davon erfahre, wisse er die anderen schon. Und genau da ist es, das Zitat: $i k$ (und dann kommt ein Zeilenumbruch in der Handschrift) mideo dreuuet. So wie es dasteht, ist das nicht $\mathrm{zu}$ verstehen, und deshalb machen die Ausgaben daraus meist ik mi de odre ииеt (wörtlich: »Ich weiß mir die anderen«). Das kann man verstehen. Doch selbst wenn man die unverständliche Worttrennung rückgängig gemacht hat, sticht der Dativ ins Auge oder die Lautformen: $i k$ ist so niederdeutsch wie uuet - aber das in einem Text, der auch hochdeutsche Formen enthält und seltsame Hybride wie heittu (was irgendwie eine Mixtur aus hochdeutsch heizu und niederdeutsch hètu ist, also ohne Lautverschiebung, aber mit Diphthong). Genau das sind die Dinge, die in der Forschung zum Hildebrandslied intensiv diskutiert wurden. Warum dieses Beispiel? - Es führt mich zu den zwei Punkten, um die es mir in diesem Essay gehen soll und die aufeinander aufbauen.

\section{Ich weiß mir die anderen ...}

Hildebrands Welt ist klein. Nach 30 Jahren kehrt er zurück, erkennt zwar seinen eigenen Sohn nicht, aber könnte ihn - wie er selbst sagt - durch die Nennung nur eines Verwandten einordnen. Er fragt nach der Sippe, weil Hildebrand nicht anonym kämpfen kann - es kommt darauf an, wer ihm gegenübersteht, und das Gegenüber wird dabei als Teil einer Gemeinschaft gesehen, für die es einsteht. Das liegt nicht nur an der Logik der Situation, denn das Lied erzählt wohl von einem Stellvertreterkampf, sondern auch genereller an den Regeln einer Kultur kleiner interagierender Eliten. Solche Eliten prägen indes nicht nur Herrschaft und Krieg, sondern auch Gelehrsamkeit und Kunst. Stefan Sonderegger hat es mehrfach gezeigt: Im Grunde gibt es nicht wirklich viele Orte und Personen, die am Spiel der frühmittelalterlichen deutschsprachigen Textüberlieferung beteiligt sind. Das Netz umspannt so gut wie den gesamten deutschen Sprachraum, aber es gibt nicht viele Knotenpunkte. Diese Netzwerke sind anders zu beschreiben als jene der sich etablierenden Funktionseliten des Hochmittelalters. Nehmen wir zwei Beispiele: Wir sind uns sicher, dass der berühmte Sangaller Codex 857, der u. a. das Nibelungenlied B und den Parzival D enthält, irgendwo im (lokal) bayerischen oder (sprachlich) bairischen Raum im zweiten Drittel des 13. Jahrhunderts entstanden ist, und wir wissen, dass er im Kontext einer Werkstatt entstand, in der man Parzival und Nibelungenlied mehrfach schrieb. Man hat aber keine Ahnung, wo diese Werkstatt genau anzusiedeln ist, was Joachim Heinzle als »kein Ruhmesblatt« bezeichnet; aber es kämen eben Dutzende von Orten in Frage! Oder: Es gehört zum festen Theseninventar der Hoch- und Spätmittelaltergermanistik, dass regionalsprachliche Uneindeutigkeiten in Handschriften 
auf eine Mixtur aus Schreibort, intendiertem Publikum, Schreiber, Autor etc. zurückgehen: Ein niederdeutsch sozialisierter Schreiber schreibe im oberdeutschen Raum für mitteldeutsche Zuhörer. So, oder so ähnlich, könnte man konstruieren und unmöglich ist da erst einmal nichts. Für die kleinen und elitären Personenkreise des Frühmittelalters dagegen sieht das anders aus! Da wechselt man weniger oft sprachgeographische Räume und wenn, dann schreibt man nicht spontan für spezifische Situationen, sondern im Rahmen fester institutioneller Zusammenhänge. Wir kennen solche Biographien: Froumund von Tegernsee, Otloh von St. Emmeram, Williram von Ebersberg oder Notker III. (den Deutschen) von St. Gallen. Und blickt man auf den prominentesten Autor der althochdeutschen Zeit, auf Otfrid von Weißenburg, dann sieht man deutlich, wie die Netzwerke bespielt werden: Er widmet sein Evangelienbuch an König Ludwig den Deutschen (seinen weltlichen Herrscher), an Liutbert von Mainz (seinen geistlichen Herrn), an Salomo I. von Konstanz (seinen Lehrer) und an Hartmuot und Werinbert (die St. Galler Mönche, Mitbrüder und wohl Mitschüler). Von Notker dem Deutschen wissen wir den Sterbetag und seine letzten Worte - und das aus der Feder seines Schülers Ekkeharts IV. von St. Gallen, dessen Hand wir in vielen Manuskripten identifizieren können, so wie wir auch Otfrids eigene Hand bei der Korrektur seines Werkes beobachten können (zumindest höchstwahrscheinlich). Vom Ezzolied - um ans Ende des Frühmittelalters zu sehen - kennen wir den Autor, den Melodiedichter, den Auftraggeber und (auf den Tag genau) den Anlass für den Auftrag. Dagegen ist das, was wir über die Stars des Hochmittelalters, was wir über Walther, Wolfram oder gar Hartmann wissen, ein Witz.

Die althochdeutschen und frühmittelhochdeutschen Texte sind - wie Ernst Hellgardt gezeigt hat - gar nicht so häufig anonym, wie das in späterer Zeit gerade geistliche Gebrauchstexte sein werden. Ambitionen, frühmittelalterliche Texte konkret in die uns bekannten institutionellen Zusammenhänge zu rücken und an Orte und Personen zu binden, sind weit weniger kühn, als das für das spätere Mittelalter der Fall sein wird.

Nehmen wir ein aktuelles Beispiel: Im Kloster Admont wurde ein neues und sehr frühes Fragment aus der Abrogans-Tradition gefunden, das ca. 50 deutsche Wörter enthält. Zur Erschließung dieses Fragments wurde ein Workshop mit 12 ExpertInnen im Kloster Admont organisiert und sogar der Spiegel berichtete - wenn auch etwas kurios - über den Fund. Für solche Effekte bräuchte man aus dem Hochmittelalter schon eigenhändige Zeilen von Wolfram, den Prolog des Erec oder ein Nibelungenlied aus dem 12. Jahrhundert - aber das ist vielleicht nur eine Frage der Performance. Es ist hier aber auch eine methodische Differenz greifbar: Fragmente des Hoch- und Spätmittelalters reihen sich meist ein in ein dichtes Netz von Daten, sind eher ein Stück Heu auf einem Heuhaufen oder ein Puzzleteil in einem Puzzle, das trotz vieler kleiner Lücken doch schon erkennen lässt, was das Puzzle als Ganzes darstellen wird. Ein Schnipsel mit Abrogans-Wortschatz dagegen ist Teil eines Puzzles, von dem erst wenige Teile zusammengesetzt sind, und wir wissen, dass der Großteil an Puzzlestücken nie mehr auftauchen wird.

Um nicht falsch verstanden zu werden: Auch im Hochmittelalter kann man nach Autoren, Orten, Zeiten und Situationen der Textentstehung fragen - und man hat das intensiv und durchaus auch radikal spekulierend getan. Aber die Nullhypothese, von 
der man ausgeht, ist für das Frühmittelalter anders konfiguriert. Es geht nicht darum, etwas aus vielen positiven Spuren zu schließen, sondern das Wenige wird mit dem Wenigen kombiniert: Nur wenn es Gegenargumente gibt, wird man annehmen, dass ein altsächsischer Großtext aus der ersten Hälfte des 9. Jahrhunderts nicht in Fulda entstanden ist.

Die losen Enden der Quellen des Frühmittelalters liegen weiter auseinander, aber weil es wenige Quellen sind, sind alle irgendwie wichtig, und die Lizenzen, die losen Enden zu verbinden, sind größer als in späteren quellenstrotzenden Zeiten. Das ist letztlich nicht nur ein statistischer Sachverhalt; die lockere Quellenlage entspricht in gewisser Hinsicht auch der Welt des Frühmittelalters, die eine Kultur der Wenigen ist. In pragmatischer Hinsicht verlangt dieses Wenige nach einer Komplettheit (nicht des Gewesenen, aber des Erhaltenen), die in späteren Zeiten nicht mehr zu haben ist. Der Handschriftencensus, ein wunderbares Hilfsmittel unseres Faches, führt das vor: Vom 8. bis zum 12. Jahrhundert sind alle deutschsprachigen Texthandschriften aufgenommen (so wie auch alle Glossenhandschriften des Frühmittelalters kataloghaft erfasst sind). Danach komplexisieren sich die Fragen: Kann man alles aufnehmen? Für das 15. Jahrhundert wenigstens werden wir das wahrscheinlich nicht mehr erleben. Aber jenseits der Quantität steht etwa die Frage, was eine deutschsprachige Handschrift denn ist: Wie viel Deutschsprachiges muss sie enthalten? Ein Wort? Eine Zeile? Einen kurzen Text? Mehrere kurze Texte? Texte von mehr als einer Seite? Prominente Texte (also einen Vers Walthers schon, aber nicht drei Zeilen Gebetsprosa)? Diese Fragen stellen sich für das Frühmittelalter nicht: Die Welt der Überlieferung ist für die Frühmittelalterforschung also karg, aber dafür klar. Karstlandschaft steht gegen Regenwald.

Ist eine Folge daraus, dass man im Bereich des Frühmittelalters mehr spekulieren darf? Im engeren Sinne nicht, denn die deutenden Schritte sind dieselben, die auch für das Verständnis des späteren Mittelalters gelten. Also: Erst wenn man >alle< wichtigen Quellen berücksichtigt hat, kann man die bestmögliche Aussage treffen (über die man aber natürlich trotzdem streiten kann). Für das Frühmittelalter ist das >alle< aber irgendwie erreichbarer und tatsächlich oft schnell erreicht; für das spätere Mittelalter rückt das >alle< in immer weitere Ferne und die Frage ist, was man berücksichtigen muss, was man berücksichtigen kann und wann man genug berücksichtigt hat. Es herrscht eine Hermeneutik der Lücke, die man lassen muss, weil man nicht alles kennen kann; für das Frühmittelalter ist die Lücke zwischen den Quellen eine Gegebenheit. Auch wenn man alles kennt, kann das Schließen dieser Lücke natürlich auch auf Abwege führen.

Drei Beispiele dazu: Am Ende des Georgsliedes, das als Nachtrag in einer Handschrift von Otfrids Evangelienbuch steht, die Wörter Nequo Wisolf. Die sind zwar von zwei Händen geschrieben, die wiederum nichts mit der Hand des Georgsliedes zu tun haben, und auch vom Layout her gibt es keine Verbindung der Wörter zum deutschen Text. - Wolfgang Haubrichs hat das alles als Beispiel disziplinärer Mythenbildung beschrieben. Doch die Versuchung war zu groß, um nicht zu folgender Deutung zu gelangen, die immerhin noch im Lexikon des Mittelalters ihr Unwesen treibt: Ein Schreiber Wisolf habe das Lied nicht fertig geschrieben, sondern mit einem Stoßseufzer (nеqueo - ich kann nicht mehr) seine Arbeit eingestellt - was 
exzellent zur katastrophalen Orthographie des Textes passt, die wirklich auf einen überforderten Schreiber hinweisen könnte.

Das ist also kaum vero, aber trotzdem so ben trovato wie eine Namensform in derselben Handschrift: Am Rande von Otfrids Evangelienbuch steht mit Griffel eingeritzt Hicila diu scona min filu las. Volker Schupp hat mit Hinweis auf Johanne Autenrieth gezeigt, dass das $H$-nur als $H$ - und nicht als $K$-gelesen werden kann, wie man das früher tat. Trotzdem transkribiert man oft noch als $K$ - und liest Kicila als Namensform der Kaiserin Gisela, die dann als Otfrid-Leserin verewigt wäre. Beide $>$ Mythen $<$ sind eigentlich falsifiziert, aber sie treffen doch den historischen Kontext einer prekären Schreibsituation und der Herrscher(innen)nähe der althochdeutschen Bibelepik.

Und ein letztes Beispiel: Die Handschrift, in der das Muspilli nachgetragen ist, wurde von Bischof Adalram von Salzburg Ludwig dem Deutschen gewidmet, also dem Deutschen, der als Initiator vieler deutschsprachiger Texte galt und gilt und der eine Art volkssprachige Kulturpolitik verfolgt habe. Was lag da näher, als daran zu denken, die unruhige Nachtragshand, die den deutschen Text schrieb, sei die des Königs selbst? Kurz: Schnell kann man vielleicht einen spekulativen Schritt zu weit gehen und die Versuchung ist groß, aus wenigen Indizien zu viel zu machen. Aber man muss eben diese Schritte tun, um überhaupt etwas sagen zu können. Man muss etwas wagen und kann dabei schneller aus der Deckung gehen als mit Thesen über die Heimat Walthers von der Vogelweide, ohne gleich populärwissenschaftlich zu wirken.

Wenn man mich nach einer pointierten Beschreibung der Differenz zwischen Früh- und Spätmittelalterforschung fragen würde, wäre die Antwort: Die Frühmittelalterforschung muss alles kennen und sich den Rest dazu denken. Die Spätmittelalterforschung kann nicht alles kennen, sie muss auswählen und dabei sehr vorsichtig sein, nicht vorschnell von dem einen auf das andere zu schließen.

\section{Ich weiß ...}

Was uns das anfängliche Beispiel ik me de odre wet aber auch zeigt, ist eine Abweichung von der handschriftlichen Form. So sehr das Manuskript des Hildebrandslieds eine heilige Kuh der Altgermanistik ist, so wenig kann ich mir vorstellen, dass sich jemand länger mit einem Wort wie mideo oder dreuиet herumschlagen würde. Sicher, Fragen etwa, wie die korrupte Worttrennung zustande kommt, welche Sprache der Schreiber im Kopf gehabt haben könnte oder ob er gar nicht mitdachte? Das sind Fragen, die man stellen könnte und stellte, aber kaum käme es zu jenem Kniefall vor der überlieferten Form, der gerade in den historischen Philologien sein Unwesen treibt. Das Abkupfern von (selbst sehr späten) Handschriften, das Nebeneinanderstellen mehrerer derselben, das den Leser ratlos und das Zitieren eines Textes unmöglich macht, das alles kennt die Frühmittelalterforschung weniger. Gut: Die Handschriftennähe gilt als sicherer Hafen gegenüber den Exzessen einer Rekonstruktionswut, die es auch in der Frühmittelalterforschung gab. Auch die Frühmittelalterforschung kennt jene tautologischen Formen handschriftennaher oder sogar handschriftensimulierender Textwiedergabe (und ich selbst war ein großer Fan 
solcher Übungen). Aber eben jene Übungen zeigen uns inzwischen doch deutlich, dass sie uns eben keine Informationen geben, die nicht in jedem Digitalisat besser zu haben wären.

Es gehört zum Alltagsgeschäft der Frühmittelaltergermanistik, dass sie sich mit Endprodukten philologischer Arbeit herumschlagen muss und dass jede Arbeit immer auch ein Weiterarbeiten an einem Prozess solcher philologischer Aneignung ist. Die Handschrift ist dabei der unhintergehbare Ausgangspunkt und blieb es für das Frühmittelalter immer, während man für spätere Zeiten heftiger von dieser Basis abrückte. Nie aber sollte die Handschrift der Endpunkt sein und hier habe ich den Eindruck, dass es darüber für spätere Zeiten keinen Konsens mehr gibt.

Eine historische Wissenschaft, meine ich, muss rekonstruieren. Sie kann das wild tun, sie kann das vorsichtig tun, aber sie kann es nicht bleiben lassen, sonst ist sie keine Wissenschaft. Gerne rümpft man die Nase vor überkommenen historistischen Ambitionen, vor einer historistischen Sehnsucht nach dem »eigentlich Gewesenen«. Das hat sicher auch seine Berechtigung, denn die Vergangenheit ist unwiederbringlich vergangen, das Gewesene (das eigentliche wie das uneigentliche) ist uns entzogen, aber das darf nicht heißen, dass wir vor diesem Zustand die Achseln zucken oder behaupten, dass das materiell Überlieferte die Grenze dessen ist, was wir über die Vergangenheit sagen können. Man kann selbstredend fragen, wann man eingreifen darf, wann man es muss, und es ist vielleicht Konsens, dass es dabei auf die Qualität ankommt, auf die Methode; Konsens ist sicher, dass man es zu weit treiben kann, aber es sollte eben auch Konsens sein, dass man es nicht lassen kann. Vergleicht man das mit den aktuellen Zugriffen auf das Hochmittelalter, dann ist die Lage für das Frühmittelalter deutlich entspannter. Man muss keinen Ereck lesen (so verdienstvoll der auch sein mag), sich in Walther-Fassungen verlieren (und dabei alles mögliche sehen, nur keine Lyrik) oder seekrank bei der Lektüre von Synopsen werden.

Wieder auf den Punkt gebracht: In der Frühmittelaltergermanistik lassen sich die Zugänge weniger leicht entkoppeln, als das für die spätere Zeit der Fall ist: Narratologische, kulturwissenschaftliche und weiß Gott welche Zugänge sind gebunden an eine strikt philologische Arbeit. Was da steht und wie es zu verstehen ist, ergibt sich aus dieser Arbeit selbst und ohne dieselbe existiert es nicht. Es ist eben etwas anderes, einem frühneuhochdeutschen Text Sinn abzugewinnen, auch wenn er die Umarbeitung eines mittelhochdeutschen Textes ist - syntaktisch kann man vieles oder alles (und oft in Einklang mit den Grammatiken) für erlaubt erklären und den Wortlaut bewältigt man dann schon irgendwie aus seinem neuhochdeutschen Sprachgefühl heraus.

Für das Althochdeutsche sind schon frühneuzeitliche Zeugnisse historisch. Während man mittelhochdeutsche Texte in der Frühen Neuzeit umschreibt und sprachlich anpasst, werden althochdeutsche Glossare und Texte bereits quasi philologisch behandelt und vor allem sprachlich kaum angetastet. Schon im Mittelalter baut sich eine Hürde zwischen den alten und den neuen Texten auf: Während Hartmann mittelhochdeutsch dichtete, schrieben zeitgleich Mönche althochdeutsche Glossen ab und machten daraus meistens keine mittelhochdeutschen. Und selbst als Hans Ried seinen Hartmann fleißig diphthongierte, druckte man bald althochdeutsche Glossen als althochdeutsche Glossen und tastete den Sprachstand nicht an. Den Texten 
des Frühmittelalters näherte man sich schon in der Frühen Neuzeit mit einer historischen (fast könnte man sagen: wissenschaftlich-philologischen) Distanz, mit der hoch- und spätmittelalterlichen Literatur stand man dagegen in einem Kontinuum des Weiterschreibens und Umarbeitens. Das ist eine wichtige Differenz, die es der Frühmittelalterforschung erspart, etwa mittelhochdeutsche Texte rückübersetzen zu müssen (oder sich darüber zu streiten, ob man das tun sollte).

Die frühmittelalterlichen Texte sind Relikte in einem Meer des Vergessens und meist ganz eigene Dinge. Natürlich sind auch in späterer Zeit alle Fälle spezielle, aber - um es wieder auf eine Formel zu bringen - im Frühmittelalter sind alle Fälle in allen Fällen spezielle. Ich habe mich einmal an eine Anthologie althochdeutscher Texte gewagt und dabei in jedem Fall überlegen müssen, wie ich editorisch vorgehe. Ich konnte mich dabei nicht einfach für eine editorische Methode entscheiden, sondern musste immer auch daran denken, was mit der Edition jeweils angestellt werden sollte. Für eine Anthologie ging es dabei meistens um die Ermöglichung von Lektüre, aber es gibt auch andere Fluchtpunkte: den Blick auf die Schreibsprache, den Schreibprozess, die Metrik, die Quellen.

Die oben angedeutete Elitenkultur spiegelt sich auch in der Schreibpraxis wider: Man weiß, was man schreibt, man schreibt für ganz spezifische Formen der Textpraxis - und was dabei herauskommt, sind wilde Orthographien, sind Kürzungen, ist die Verwendung alternativer Schriftformen (wie etwa Geheimschriften oder fremde Schriftzeichen). Die kann man nicht editorisch erschließen, indem man sie einfach wiedergibt: Eine Zeile der Murbacher Hymnen würde nach der Handschrift so aussehen: folliu sint la ti da (XXVI,3). So steht es da. Nun finden sich diese deutschen Wörter zwischen lateinischen Zeilen und zwar über dem Syntagma Pleni sunt caeli et terre, und das zeigt uns, dass la ti da dabei caeli et terre übersetzen muss. Die Ausgaben ergänzen also: (himi)la (in)ti (er)da. Es stehen hier also nur die Endungen und die Ausgabe ergänzt das, was man sich dazudenken muss. Dazudenken kann man es sich natürlich nur mit Blick auf das Lateinische, sodass wir hier gar keinen deutschen Text vor uns haben, sondern ein Ensemble aus Latein und Deutsch, das nur als Ganzes Sinn ergibt. Sicher: Neophilologen hätten recht mit der Behauptung, dass es den Text himila inti erda nie gegeben habe - er entspringt aber nicht der Phantasie der Philologen, sondern der Frage, was die sonst sinnlosen Buchstaben denn eigentlich bedeuten könnten und was die Benutzer der Handschrift gelesen oder verstanden haben könnten. Dabei kann man sicher mal daneben liegen, aber wenn das dazu führen würde, dass man la ti da liest, dann kann man sich zumindest ein Studium sparen. Sicher: Für hoch- und spätmittelalterliche Zeiten war es nötig, das Ruder einer ahistorischen Rekonstruktionswut herumzureißen, für das Frühmittelalter musste man das nicht so drastisch einfordern, weil die Praktiken der editorischen Textarbeit viel deutlicher im fachlichen Bewusstsein blieben und als gemeinsame Aufgabe präsent sind. Jeder weiß, dass man sich so oder so entscheiden kann und man liest die Texte als Ensemble von handschriftlicher Form, philologischer Entscheidung und editorischer Umsetzung - und nicht als Reflex einer Sehnsucht nach einem Original. Es scheint mir, dass die aktuelle Philologie des Hoch- und Spätmittelalters hier von der Philologie des Frühmittelalters lernen sollte. Ich denke, dass man langsam aufhören sollte, die Beerdigung von goethezeitlichen, genieästhetischen Autor- und Werkvorstellungen euphorisch zu feiern. Beerdigungen sollten 
irgendwann zu Ende sein, Toten sollte man ihre Ruhe gönnen. Offen gesagt glaube ich, dass es sich wohl bis in die letzten Winkel herumgesprochen hat, dass man da auf dem Holzweg war. Jetzt sollte es darum gehen, wie wir mit Autoren und Werken umgehen, die unter anderen kulturellen Prämissen zu denken sind, und uns nicht in die Arme lustloser Abschreiber werfen: Deren Produkte unhinterfragt zu nobilitieren, ist genauso absurd, wie nach einer originalen Autorintention zu fragen.

\section{Fazit}

Sherlock also gegen Big Brother - der, der aus Wenigem das Bild eines Ganzen macht, gegen den, der das Ganze gleichzeitig sehen will und trotz der Unmöglichkeit dieser Wahrnehmung hofft, das Entscheidende zu finden - und doch oft gerade das nicht tut. Aber macht es wirklich Sinn, die beiden Bereiche so euphorisch zu trennen, wie ich das hier für ein Heft der LiLi getan habe? Ja, ich denke schon, und sei es nur darum, dass man voneinander lernt. Philologie ist Wissenschaft, aber sie ist auch Habitusform. Es geht um den Umgang mit den geliebten Wörtern und um die Positionierung gegenüber denselben. Da kann es keine Generalformel geben, aber mir scheint es wichtig zu sein, sich nicht zu sehr aus diesem Spiel herauszunehmen! Texte sind nicht einfach so, wie sie sind, sie sind gemacht und auch handschriftliche Fassungen sollte man nicht einfach als gegeben hinnehmen und sich vom Bild der Codices nicht einschüchtern lassen, so eindrucksvoll es uns auch in Form von Digitalisaten entgegentritt. Die Texte des Frühmittelalters verlangen uns das vielleicht deutlicher ab, sonst blieben sie stumm, aber auch die Texte späterer Zeiten, selbst wenn sie deutlicher in einem sprachlichen und literarischen Kontinuum mit uns selbst stehen, warten auf einen philologischen Zugriff.

Um nicht falsch verstanden zu werden: Ich rede keiner althergebrachten Lachmann-Philologie das Wort, die irgendwelchen Originalen nachjagt und dabei anachronistisch ein Phantasma der Moderne auf das Mittelalter zurückprojiziert. Aber! Und das ist ein sehr großes ABER! Eine Philologie, die das Bild der Handschrift zum unhintergehbaren Faktum erklärt, verliert vieles. Auch ohne >Original< und ohne genieästhetische Autorvorstellungen fielen Texte nicht von den Bäumen und haben sich Menschen irgendetwas beim Verfassen von Texten gedacht. Man muss nicht genau wissen, was eigentlich, und man muss deren Produkte nicht gleich als gut oder schlecht bezeichnen, aber es scheint mir zu defensiv zu sein, nur dann in das handschriftliche Bild einzugreifen, wenn das gar keinen Sinn mehr ergibt (vor allem wenn man mit dem, was man als sinnvoll akzeptiert, in jüngster Zeit wirklich sehr, sehr großherzig geworden ist). Handschriften sind Handschriften, Texte sind Texte, sie wurden einst geschrieben und gelesen und sie sind jetzt Gegenstand der Philologie. Wie verhalten sich diese Dimensionen zueinander? Wenn man nicht vergisst, darauf Antworten zu suchen, wird alles gut.

Open access funding provided by University of Vienna.

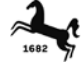


Open Access Dieser Artikel wird unter der Creative Commons Namensnennung 4.0 International Lizenz (http://creativecommons.org/licenses/by/4.0/deed.de) veröffentlicht, welche die Nutzung, Vervielfältigung, Bearbeitung, Verbreitung und Wiedergabe in jeglichem Medium und Format erlaubt, sofern Sie den/die ursprünglichen Autor(en) und die Quelle ordnungsgemäß nennen, einen Link zur Creative Commons Lizenz beifügen und angeben, ob Änderungen vorgenommen wurden. 